\title{
Effects of blood oxygen saturation on pulmonary artery remodeling in an in vitro perfusion circuit model
}

\author{
Yao Liu, Bin Wen, Fengwen Zhang, Xiang-Bin Pan \\ Department of Cardiovascular Surgery, National Center for Cardiovascular Disease, China \& Fuwai Hospital, Chinese Academy of Medical Sciences \\ \& Peking Union Medical College, Beijing, China \\ Contributions: (I) Conception and design: XB Pan; (II) Administrative support: XB Pan; (III) Provision of study materials or patients: Y Liu, B Wen; (IV) \\ Collection and assembly of data: Y Liu, B Wen; (V) Data analysis and interpretation: Y Liu, B Wen, F Zhang; (VI) Manuscript writing: All authors; \\ (VII) Final approval of manuscript: All authors. \\ Correspondence to: Xiang-Bin Pan. National Center for Cardiovascular Disease, China \& Fuwai Hospital, Chinese Academy of Medical Sciences \& \\ Peking Union Medical College, 167 Beilishi Road, XiCheng District, Beijing 100037, China. Email: panxiangbin@fuwaihospital.org.
}

Background: Patients with transposition of the great arteries are likely to survive surgery despite severe pulmonary artery hypertension. However, the underlying mechanisms remain largely unknown. The present study aimed to test the hypothesis that high blood oxygen saturation may protect the pulmonary artery from remodeling.

Methods: An in vitro pulmonary artery perfusion model was successfully performed by connecting rabbit pulmonary artery to a closed perfusion circuit. Twenty-five rabbits were divided randomly into 5 groups according to exposure conditions: Normal Control (NC) group (unperfused normal pulmonary artery), High Saturation (HS) group (oxygen saturation range: 90-100\%), Medium Saturation (MS) group (oxygen saturation: 65-75\%); Low Saturation (LS) group (oxygen saturation: 40-50\%), and anti-hypoxia inducible factor- $1 \alpha$ (anti-HIF-1 $\alpha$ ) group (oxygen saturation range: 40-50\%, and LW6, which is a novel HIF- $1 \alpha$ inhibitor; was added). By staining and optical microscopy examination, pathological morphology was analyzed, and the protein expression levels of HIF-1 $\alpha$, angiotensin-II (Ang-II), endothelin-1 (ET-1), Rhoassociated protein kinase-1 (Rock-1), and matrix metallopeptidase-2 (MMP-2) were determined by Western blotting.

Results: The amounts of elastin, muscle, and collagen and the protein levels of ET-1, HIF-1 $\alpha$, Rock-1, and MMP-2, increased significantly with decreased oxygen saturation in the perfusion circuit. A significant improvement in pathological morphology was observed in the anti-HIF1 $\alpha$ group. The expression of HIF-1 $\alpha$, ET-1, Ang-II, Rock-1, and MMP-2 in the anti-HIF1 $\alpha$ group was also significantly lower than that in the LS group.

Conclusions: In the closed perfusion model, high blood oxygen saturation alleviated pulmonary vascular structural remodeling. Similar beneficial effects were observed when inhibiting the HIF-1 $\alpha$ protein, suggesting a key role for HIF- $1 \alpha$ in pulmonary artery remodeling.

Keywords: Hypoxia inducible factor-1 $\alpha$ (HIF-1 $\alpha)$; pulmonary artery; hypertension; in vitro perfusion; oxygen saturation

Submitted Jun 12, 2020. Accepted for publication Feb 06, 2021.

doi: $10.21037 /$ jtd-20-2124

View this article at: http://dx.doi.org/10.21037/jtd-20-2124

(c) Journal of Thoracic Disease. All rights reserved. 


\section{Introduction}

Pulmonary hypertension $(\mathrm{PH})$ is a relatively common complication of congenital heart disease and presents at any age from infancy to adulthood (1). Transposition of the great arteries (TGA) is associated with persistent $\mathrm{PH}$, with a reported incidence of $1-3 \%$ of neonates born with TGA (2). The combination of $\mathrm{PH}$ and TGA often results in poorer outcome and prognosis (3).

However, there was a peculiar phenomenon in patients with TGA and ventricular septal defect (VSD) $(4,5)$. Patients with TGA/VSD and severe PH could have improved quality of life after surgery (4-6). Yamaki et al. also found the pulmonary arterial media was significantly thicker in VSD alone than in TGA/VSD (7). In TGA patients, the pulmonary artery receives fully oxygenated blood oxygen saturation (nearly $100 \%$ ) from the left ventricle $(8,9)$. Therefore, we established an in vitro closed-circuit model to test the hypothesis that different oxygen saturation levels might lead to different extents of pulmonary vascular remodeling in a high-pressure perfusion environment and define the possible underlying mechanisms. We present the following article in accordance with the ARRIVE reporting checklist (available at http://dx.doi.org/10.21037/jtd-202124).

\section{Methods}

Twenty-five male New Zealand white rabbits (weight: $3.0 \pm 0.5 \mathrm{~kg}$ ) were obtained from Beijing Vital River Laboratory Animal Technology (Beijing, China). All animals received humane care in compliance with the "Guide for the Care and Use of Laboratory Animals" prepared and formulated by the Institute of Laboratory Animal Resources and published by the National Institutes of Health (NIH Publ No. 86/23, 1985). The protocol was approved by the Institutional Animal Care and Use Committee of Fuwai Hospital [Ethicsethics approval number:No. 0086-4-36-GZ(Z)]. Laboratory rabbits were individually housed in cages with a 12:12-h light-dark phase at a standard temperature $\left(21 \pm 1{ }^{\circ} \mathrm{C}\right)$ and controlled humidity $(50 \% \pm 10 \%)$.

The 25 rabbits were randomly divided into 5 groups ( $\mathrm{n}=5$ /group) as follows: Normal Control (NC) group (unperfused normal pulmonary artery), High Saturation (HS) group (oxygen saturation range: 90-100\%), Medium Saturation (MS) group (oxygen saturation: 65-75\%); Low Saturation (LS) group (oxygen saturation: $40-50 \%$ ), and
anti-HIF1 $\alpha$ group (oxygen saturation range: $40-50 \%$, and LW6, which is a novel HIF-1 $\alpha$ inhibitor; $20 \mu M$, Merck Millipore, Darmstadt, Germany; was added).

\section{Organ culture}

After overnight fasting, the rabbits were anesthetized by intravenous injection of $30 \mathrm{mg} / \mathrm{kg}$ pentobarbital sodium (Merck, Darmstadt, Germany). Procedures and instruments were following aseptic technique. After sternotomy, systemic heparinization (125 U/kg) was achieved. Pulmonary artery cannulation was then performed. Blood was collected via the inferior vena cava. To set the oxygen saturation and flow rate independently, each arterial segment was connected to a perfusion circuit (Figure 1). The closed perfusion circuit consisted of a reservoir, a peristaltic pump (Masterflex L/S, model: 7725062; ColeParmer, Vernon Hills, IL, USA), an oxygenator membrane, a blood oxygen saturation monitor (Medtronic Biotrend, Minneapolis, USA), and a pressure monitor (Siemens Sirecust, Munich, Germany) allowing for the application of controlled intraluminal oxygen saturation. Vessel segments were immersed in culture medium, consisting of Dulbecco's modified Eagle's medium (Gibco BRL, Grand Island, NY, USA) and antibiotics (100 IU/L penicillin, $100 \mathrm{mg} / \mathrm{L}$ streptomycin, and $10 \mathrm{~g} / \mathrm{L}$ fungizone), supplemented with $5 \%$ fetal calf serum (Boehringer Mannheim, Mannheim, Germany) (10,11).

Before cannulation, the circuit was primed with $50 \mathrm{~mL}$ rabbit whole blood. The pulmonary artery segment was perfused by a roller pump (Masterflex L/S, model: 7725062; Cole-Parmer, USA). The mean pressure was maintained at $40-50 \mathrm{mmHg}$. Blood oxygen saturation and pressure were monitored and recorded. The pulmonary artery was immersed in the above culture medium and incubated for $48 \mathrm{~h}$ at $37{ }^{\circ} \mathrm{C}, 5 \% \mathrm{CO}_{2}$, and $95 \%$ humidity. A schematic diagram of the closed-circuit is shown in Figure 1.

\section{Tissue extraction}

After perfusion, the vessel segments were removed and cut into 3-4-mm long rings. Some were flash frozen in liquid nitrogen and then stored at $-80^{\circ} \mathrm{C}$. Others were immersed in a solution of $10 \%$ neutral-buffered formalin for $24 \mathrm{~h}$ at room temperature. The fixed vessel rings were placed in embedding cassettes, dehydrated in a series of ethanol dilutions, cleared with xylene, and finally embedded in paraffin. 


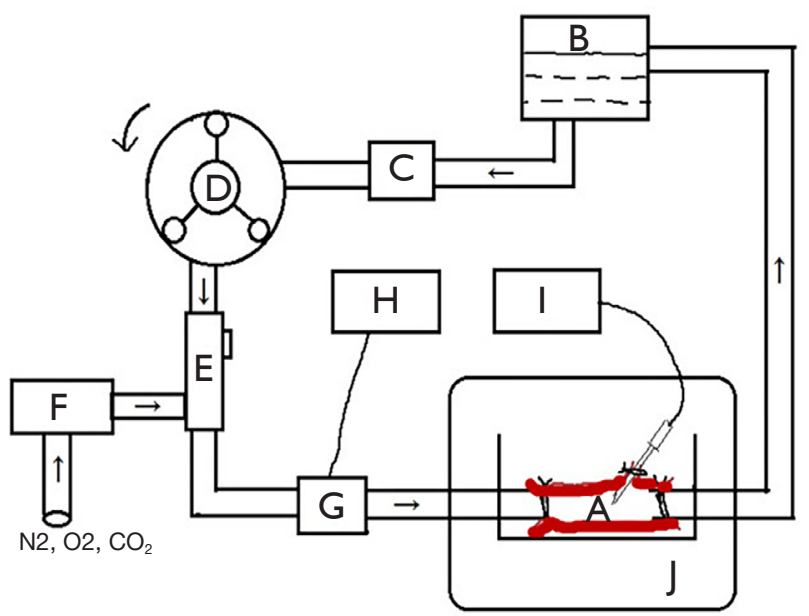

Figure 1 Closed perfusion system. Schematic drawing of the experimental setup. A motor-driven roller pump (D) pumps heparinized blood through an oxygenator membrane (E), connected to a mixed gas cylinder (F). Blood flows into the pulmonary artery (A), while the return is collected in a reservoir (B). Changes in blood oxygen saturation and pressure were monitored. (C) Bubble trap. (G) Blood oxygen saturation monitor point. $(\mathrm{H})$ BioTrend Oxygenation Saturation and Hematocrit Monitor. (I) Pressure monitor (SIEMENS Sirecust 961). (J) Thermo Scientific $\mathrm{CO}_{2}$ incubator.

\section{Morphological analysis}

Sections of $4 \mu \mathrm{m}$ were cut from each tissue block. Serial sections were stained with hematoxylin-eosin, Weigert's iron hematoxylin, and Van Gieson's solution. Microscopic examination showed black elastin, yellow muscle, and pink collagen. Image analysis was done using Image Pro-Plus 6.0 software (Media Cybernetics). The media thickness was defined as the distance between the internal and external elastic lamina. The amounts of elastin, muscle, and collagen were measured at 12 locations spaced evenly around the perimeter, and a mean value was calculated for each section. The morphological evaluation was performed independently by a pathologist who was blinded to the study.

\section{Western blot analysis}

Tissue samples (100-200 mg) were lysed with ice-cold RIPA lysis buffer. Lysates were centrifuged at $10,000 \times \mathrm{g}$ for $15 \mathrm{~min}$ at $4{ }^{\circ} \mathrm{C}$. The supernatants were collected. Protein contents were determined using Bradford protein assay.
Samples containing $30 \mu \mathrm{g}$ of protein were electrophoresed on $4 \%$ or $12 \%$ sodium dodecylsulfate-polyacrylamide gel electrophoresis and transferred onto nitrocellulose membranes (Bio-Rad). After blocking with 5\% nonfat dry milk, the membranes were incubated with anti-angiotensinII (Ang-II, Abcam EPR20599), endothelin-1 (ET-1, Abcam TRET.48.5), hypoxia inducible factor- $1 \alpha$ (HIF- $1 \alpha$, Abcam H1ALPHA67), Rho-associated protein kinase-1 (Rock1, Abcam MM1093-15D32), matrix metallopeptidase-2 (MMP-2, Proteintech 10373-2-AP), or GAPDH (Proteintech 10494-1-AP) antibodies. After washing, membranes were incubated with secondary antibodies. The antigen-antibody complexes were visualized by an enhanced chemiluminescence (ECL) system (Amersham). AngII, ET-1, MMP-2, Rock-1, HIF-1 $\alpha$, and GAPDH were identified as single bands of $51,30,72,158,92$, and $37 \mathrm{kDa}$, respectively, which were consistent with published sizes.

\section{Statistical analysis}

Statistical analysis was performed with SPSS 15.0 for Windows (SPSS, Chicago, IL, USA). All data were expressed as the mean \pm standard deviation, $\mathrm{P}<0.05$ was considered statistically significant. One-way analysis of variance (ANOVA) was used to determine differences between the 5 groups. Post-hoc least significant difference test was carried out if the initial ANOVA was significant.

\section{Results}

\section{Evaluation of the perfusion model}

The pulmonary artery perfusion model was established successfully. No blood vessel rupture or leakage occurred during the period of perfusion. The difference in blood oxygen saturation and partial pressure of oxygen among the different groups $(\mathrm{n}=5)$ was statistically significant $(\mathrm{P}<0.05)$ (Table 1). Blood oxygen saturation was following the experimental design, and the model established the gradient of oxygen saturation in the closed-circuit system.

\section{Pathological morphological analysis}

Arterial lumen enlargement, arterial wall thickening, loss of elasticity, and permeability were found in the HS, MS, LS, and anti-HIF $1 \alpha$ groups (n=5/group) (Figures 2,3). Microscopic observation revealed that pulmonary artery wall thickening mainly occurred in the media layer. The 
Table $1 \mathrm{SO}_{2}$ and $\mathrm{PaO}_{2}$ by blood oxygen saturation group

\begin{tabular}{lccc}
\hline & HS group $(\mathrm{n}=5)$ & MS group $(\mathrm{n}=5)$ & LS group $(\mathrm{n}=5)$ \\
\hline $\mathrm{SO}_{2}(\%)$ & $97.94 \pm 1.01^{\Delta}$ & $72.14 \pm 12.85^{\star}$ & $43.83 \pm 8.71^{\star \Delta}$ \\
$\mathrm{PaO}_{2}(\mathrm{mmHg})$ & $174.15 \pm 28.65^{\Delta}$ & $42.15 \pm 14.35^{\star}$ & $20.85 \pm 10.13^{\star \Delta}$ \\
\hline
\end{tabular}

Data are presented as mean $\pm \mathrm{SD}$. ${ }^{*} \mathrm{P}<0.05$ compared with $\mathrm{HS}$ group; ${ }^{\wedge} \mathrm{P}<0.05$ compared with $\mathrm{MS}$ group; $1 \mathrm{mmHg}=0.133 \mathrm{kPa}$.

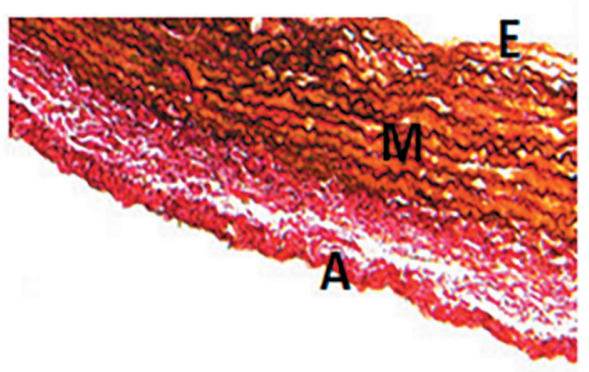

NC Group

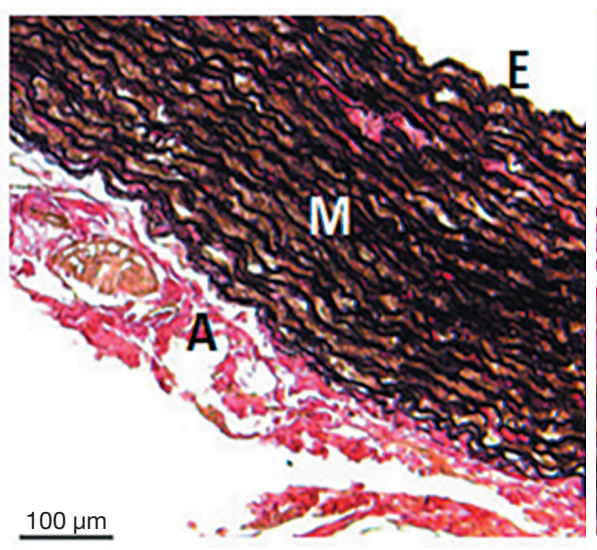

LS Group

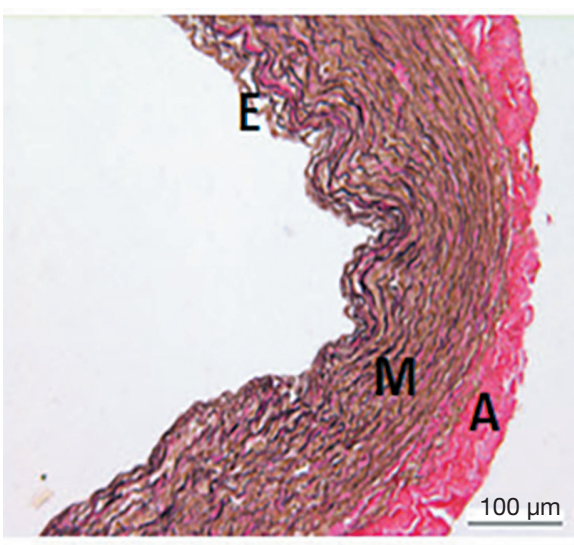

HS Group

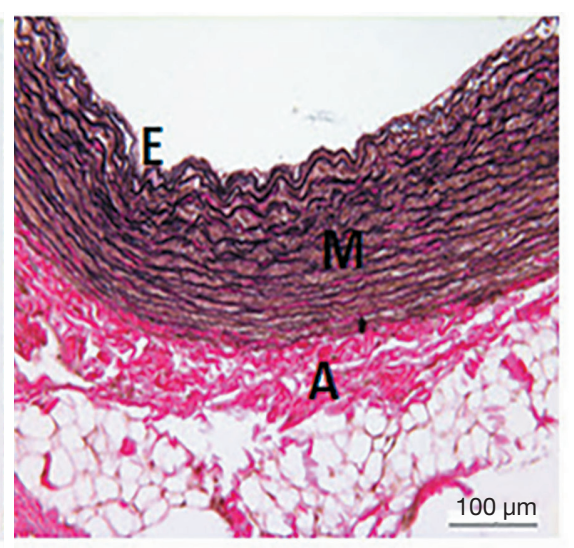

MS Group

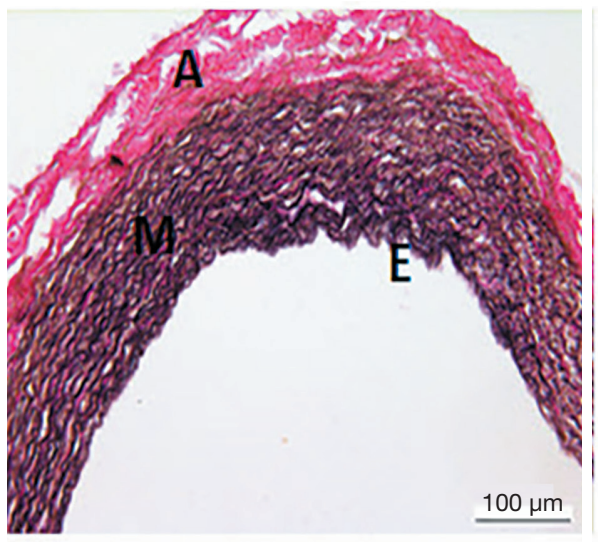

Anti-HIF1a Group

Figure 2 Photomicrographs showing a cross-section of the pulmonary artery in the 5 study groups (n=5/group). Elastin (black), muscle (yellow), and collagen (pink). A, adventitia layer; E, endothelial cells lining in the intimal layer; M, media layer.

endothelial cells lining the intimal layer were injured and even damaged. Elastin (black), muscle (yellow), and collagen (pink) content in the media layer were significantly higher in the HS, MS, and LS groups $(\mathrm{P}<0.05)$ compared with the control group. The amounts of elastin, muscle, and collagen increased significantly as blood oxygen saturation decreased. Furthermore, a significant improvement in pathological morphology was observed in the anti-HIF1 $\alpha$ group $(\mathrm{P}<0.05)$.
Expressions of HIF-1 $\alpha$, ET-1, Rock-1, Ang-II, and MMP2 in pulmonary artery tissue

Western blotting showed that the increased expressions of HIF-1 $\alpha$, ET-1, Rock-1, and MMP-2 in the pulmonary artery tissue were associated with decreased blood oxygen saturation in the perfusion system $(\mathrm{P}<0.05)$ except for Ang-II (Figure 4). The expressions of HIF-1 $\alpha$, ET-1, AngII, Rock-1, and MMP-2 in the anti-HIF $1 \alpha$ group were 

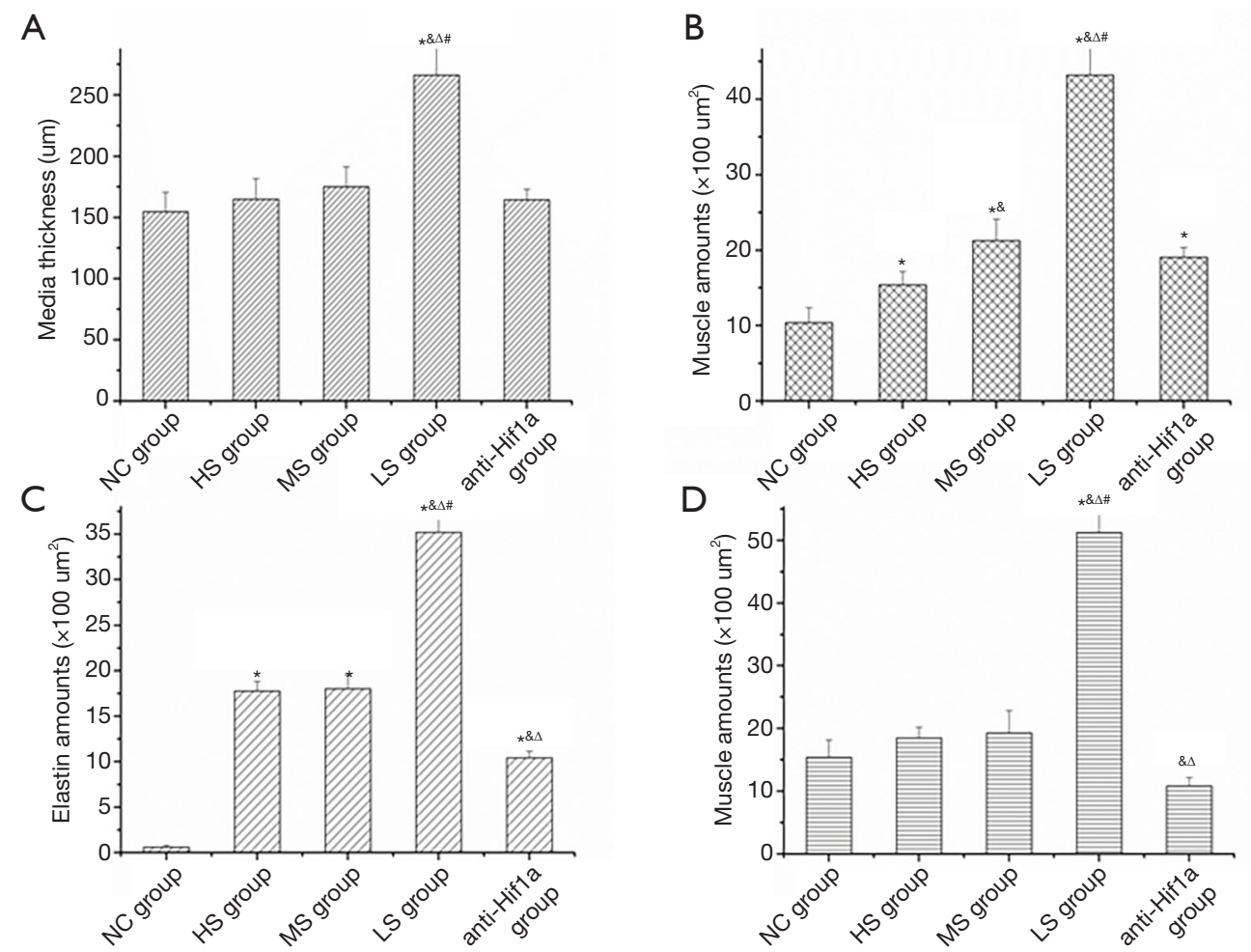

Figure 3 Pathological morphological analysis. (A) Media layer thickening. (B) Total amount of collagen content. (C) Total amount of elastin content. (D) Total amount of muscle content. ${ }^{*} \mathrm{P}<0.05$ compared with the NC group; ${ }^{8} \mathrm{P}<0.05$ compared with the HS group; ${ }^{\Delta} \mathrm{P}<0.05$ compared with the MS group; ${ }^{\#} \mathrm{P}<0.05$ compared with the anti-HIF1 $\alpha$ group. Data were evaluated by one-way analysis of variance (n=5/ group). Results are reported as means \pm standard deviations.

significantly lower than those in the LS group $(\mathrm{P}<0.05)$.

\section{Discussion}

Although many studies have documented $\mathrm{PH}$ induced by hypoxia or hyperoxia, they have only focused on the amount of oxygen inhaled, affecting oxygen saturation in the alveoli and pulmonary vein $(8,12,13)$. The effect of pulmonary artery oxygen saturation on $\mathrm{PH}$ and its underlying mechanism remains to be fully elucidated. Oxygen saturation reflects how much oxygen an individual's blood can carry. Healthy individuals exhibit pulmonary artery (PA) oxygen saturation values at $76 \%$, even in those who are given $100 \%$ oxygen to breathe. In contrast, oxygen saturation level in $\mathrm{PA}$ is almost $100 \%$ in patients with TGA. In healthy individuals, fully oxygenated blood only reaches the bronchial arteries, which then nourishes the pulmonary arteries. It remains unclear whether pulmonary arterial blood's oxygen saturation affects the remodeling of pulmonary vessels, and it is certainly worthwhile to study the protective mechanism of high oxygen saturation on pulmonary arterial remodeling in patients with TGA $(9,13)$.

The isolated closed pulmonary arterial perfusion circuit used in our study is convenient and technically achievable. The model has been used to investigate hypertensive vascular remodeling mechanisms by perfusing mouse carotid arteries in a closed circuit (14). Furthermore, our closed perfused circuit produced an in vitro model that simulates pulmonary artery hypertension with characteristic structural and functional changes in pulmonary arteries. After perfusion, the pulmonary artery exhibited arterial lumen enlargement, arterial wall thickening, loss of elasticity, and permeability. The proliferation of pulmonary arterial smooth muscle cells and elastic fiber was significantly increased, and the pulmonary artery was muscularized via media layer thickening. When the gradient of oxygen saturation was decreased, the thickness of the pulmonary artery wall, vascular smooth muscle cells, and collagen fibers increased, accompanied by the increased expressions 
A
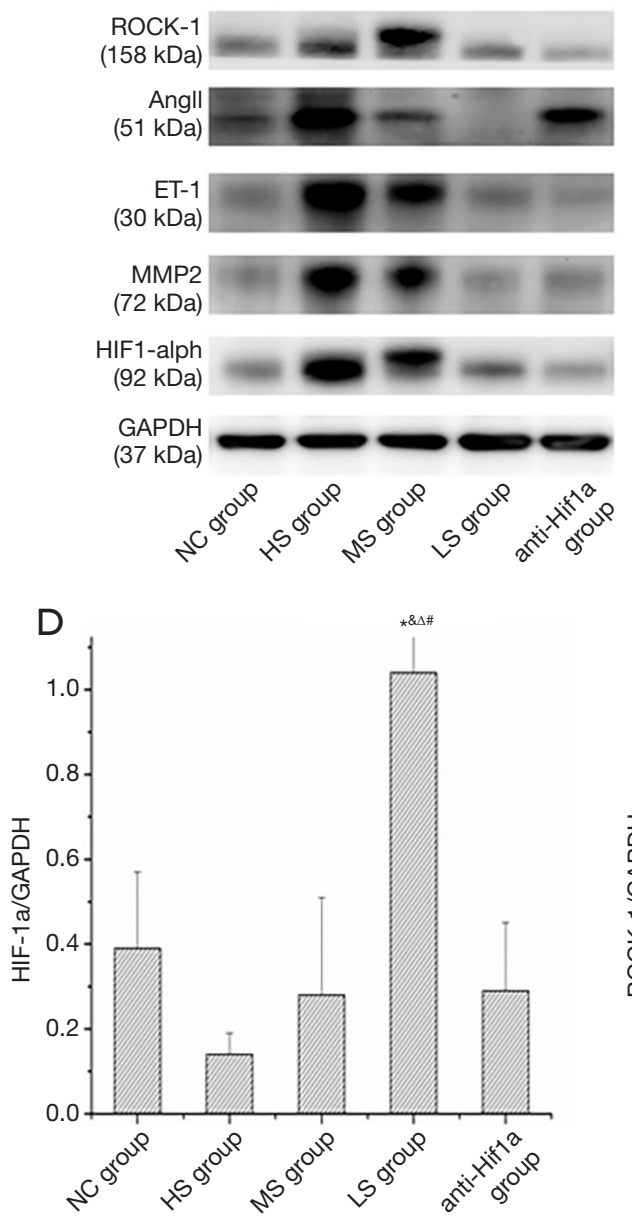

B
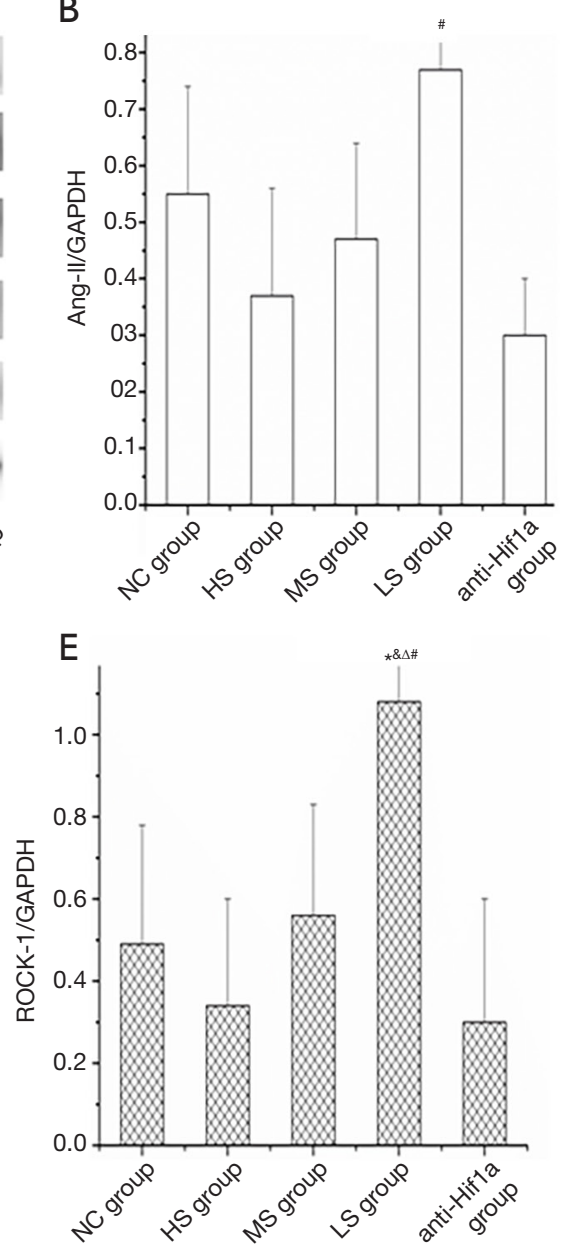

C

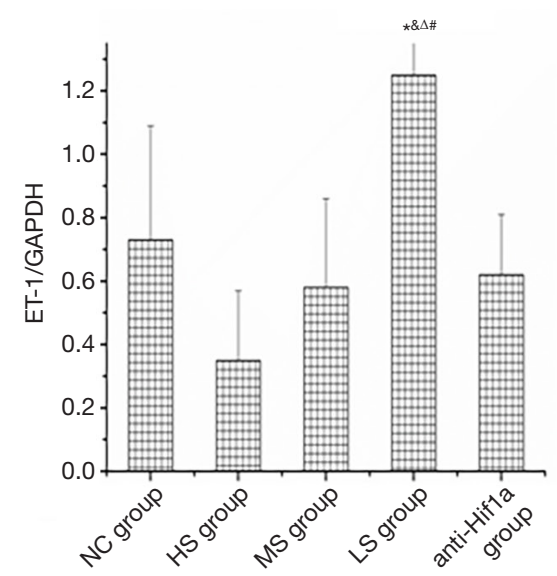

$\mathrm{F}$

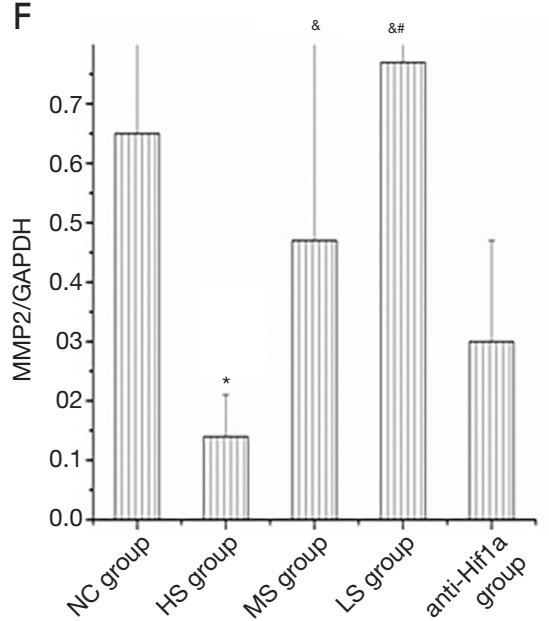

Figure 4 Western blotting (A) and quantification of expression for Ang-II (B), ET-1 (C), HIF-1 $\alpha$ (D), Rock-1 (E), and MMP-2 (F) among study groups (n=5/group). ${ }^{*} \mathrm{P}<0.05$ compared with the $\mathrm{NC}$ group, ${ }^{8} \mathrm{P}<0.05$ compared with the HS group; ${ }^{\circ} \mathrm{P}<0.05$ compared with the MS group; ${ }^{"} \mathrm{P}<0.05$ compared with the anti-HIF $1 \alpha$ group. Data were evaluated by one-way analysis of variance test. Results are reported as means \pm standard deviations.

of HIF-1 $\alpha$, ET-1, and MMP2. These factors may play an important role in enhancing pulmonary vascular contraction and remodeling.

HIF-1 is a highly conserved transcription factor that regulates the oxygen-dependent expression of hundreds of genes. It is a heterodimeric transcription factor composed of a constitutively expressed $\beta$-subunit and an oxygen responsive $\alpha$-subunit. Under hypoxic conditions, HIF- $1 \alpha$ is stabilized and translocated into the nucleus, binding to hypoxia-responsive elements, and activates the expression of genes, such as ET-1 and Ang-II $(9,13,15)$. Increased ET-1 or Ang-II level activates the RhoA/Rho kinase pathway, leading to MMP-2 and vascular smooth muscle cell hyperconstriction $(9,16)$. MMP-2 promotes vascular smooth muscle cell proliferation and pulmonary vascular remodeling $(17,18)$. Our study revealed that increased expressions of ET-1, Rock-1, and MMP-2 correlated with decreased blood oxygen saturation. Expressions of HIF$1 \alpha$, ET-1, Ang-II, Rock-1, and MMP-2 in the anti-HIF1 $\alpha$ group were significantly lower than those in the LS group $(\mathrm{P}<0.05)$. HIF-1 $\alpha$ regulates the proliferation of vascular smooth muscle cells and plays a key role in pulmonary vascular remodeling. Therefore, targeting HIF-1 $\alpha$ may improve pulmonary artery hypertension outcome.

Interestingly, there was no correlation between increased Ang-II level and decreased oxygen saturation 


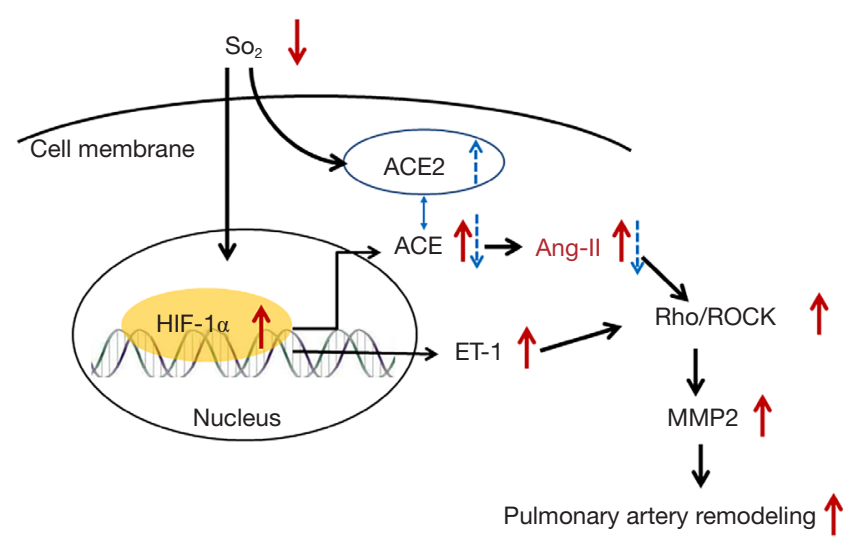

Figure 5 Schematic of hypoxia-driven regulation of angiotensinconverting enzyme and ACE2. Low oxygen saturation responses via HIF-1 $\alpha$ transactivate ACE and lead to Ang-II formation. Low oxygen saturation upregulates ACE2 levels via a HIF-1 $\alpha$ independent pathway.

value in our study, which might be explained by the more complex and context-dependent Ang-II regulation system. A previous study revealed that the upregulation of angiotensin-converting enzyme (ACE) 2 level by hypoxia might counterpoise the effect of increased ACE, which is an enzyme that converts Ang-I to Ang-II. Therefore, increased Ang-II expression was not correlated to decreased oxygen saturation (Figure 5). Hypoxia can directly increase the expression of ACE2, which may be a protective factor for the development of hypoxia $\mathrm{PH}$ in the early stage $(8,19)$.

The present study has several limitations. First, the perfusion model's major advantages are that it is easy to perform, control for confounding, and avoid certain biases. However, the body's internal environment is not so simple. It is a complex environment affected by multiple organs and systems. Therefore, high oxygen saturation's clinical application value also needs to be fully verified in in vivo experiments. How to achieve high oxygen conditions in pulmonary arteries is also the direction of future research. Second, several studies have shown that HIF-1 $\alpha$ inhibitors can effectively reduce vascular smooth muscle cell proliferation (15,17-19). Therefore, inhibiting HIF-1 $\alpha$ could become a new approach in PAH therapy. However, it is necessary to perform further studies to prevent and reduce unintended side-effects of HIF- $1 \alpha$ inhibitors in the treatment of PAH. There is still much work to be done before these inhibitors can be introduced into clinical practice. Third, the detailed mechanism requires further verification by cell culture and gene knockdown animal models. Finally, the sample size was small, and further large-sample experimental and clinical studies are required.

\section{Conclusions}

To the best of our knowledge, the present study is the first to demonstrate that high blood saturation alleviates pulmonary vascular structure remodeling in a perfusion circuit model. HIF- $1 \alpha$ plays a key role in pulmonary artery remodeling in the low oxygen saturation closed perfusion model, and the inhibition of HIF-1 $\alpha$ yielded the same effect like high blood saturation.

\section{Acknowledgments}

Funding: This work was supported by the National Natural Science Foundation of China (81670289) and National Science Foundation for Young Scientists of China (81400041).

\section{Footnote}

Reporting Checklist: The authors have completed the ARRIVE reporting checklist. Available at http://dx.doi. org/10.21037/jtd-20-2124

Data Sharing Statement: Available at http://dx.doi. org/10.21037/jtd-20-2124

Conflicts of Interest: All authors have completed the ICMJE uniform disclosure form (available at http://dx.doi. org/10.21037/jtd-20-2124). The authors have no conflicts of interest to declare.

Ethical Statement: The authors are accountable for all aspects of the work in ensuring that questions related to the accuracy or integrity of any part of the work are appropriately investigated and resolved. All animals received humane care in compliance with the "Guide for the Care and Use of Laboratory Animals" prepared and formulated by the Institute of Laboratory Animal Resources and published by the National Institutes of Health $(\mathrm{NIH}$ Publ No. 86/23, 1985). The protocol was approved by the Institutional Animal Care and Use Committee of Fuwai Hospital [ethics approval No. 0086-4-36-GZ(Z)]. 
Open Access Statement: This is an Open Access article distributed in accordance with the Creative Commons Attribution-NonCommercial-NoDerivs 4.0 International License (CC BY-NC-ND 4.0), which permits the noncommercial replication and distribution of the article with the strict proviso that no changes or edits are made and the original work is properly cited (including links to both the formal publication through the relevant DOI and the license). See: https://creativecommons.org/licenses/by-nc-nd/4.0/.

\section{References}

1. Ivy DD, Abman SH, Barst RJ,etal. Pediatric Pulmonary Hypertension. J Am Coll Cardiol 2013;62:D117-26.

2. Luciani GB, Chang AC, Starnes VA. Surgical repair of transposition of the great arteries in neonates with persistent pulmonary hypertension. Ann Thorac Surg 1996;61:800-5.

3. Kumar A, Taylor GP, Sandor GG, et al. Pulmonary vascular disease in neonates with transposition of the great arteries and intact ventricular septum. Br Heart J 1993;69:442-5.

4. Prêtre R, Tamisier D, Bonhoefer P. Results of the arterial switch operation in neonates with transposed great arteries. Lancet 2001;357:1826-30.

5. Lui RC, Zhuang J, Lei BF. A better option for patients with TGA/VSD and severe pulmonary hypertension undergoing palliative arterial switch operation. Ann Thorac Surg 2012;93:692-9.

6. Nakajima Y, Momma K, Seguchi M, et al. Pulmonary hypertension in patients with complete transposition of the great arteries: midterm results after surgery. Pediatr Cardiol 1996;17:104-7.

7. Yamaki S, Endo M, Takahashi T. Different grades of medial hypertrophy and intimal changes in small pulmonary arter-ies among various types of congenital heart disease with pulmonary hypertension. Tohoku J Exp Med 1997;182:83-91.

8. Fan H, Hu S, Zheng Z, et al. Do patients with complete transposition of the great arteries and severe pulmonary hypertension benefit from an arterial switch operation? Ann Thorac Surg 2011;91:181-6.

9. Pan X, Zheng Z, Hu S, et al. Mechanisms of pulmonary hypertension related to ventricular septal defects congenital heart disease. Ann Thorac Surg 2011;92:2215-20.

10. Wisser W, Oturanlar D, Minich R, et al. Closed circuit perfusion of an isolated rabbit lung A new model for the evaluation of preservation quality of stored lungs. Eur J Cardiothorac Surg 1993;7:71-4.

11. dos Santos PR, Iskender I, Machuca T, et al. Modified in vivo lung perfusion allows for prolonged perfusion without acute lung injury.J Thorac Cardiovasc Surg 2014;147:774-81.

12. Reddy VM, Meyrick B, Wong J, et al. In utero placement of aorta pulmonary shunts. A model of postnatal pulmonary hypertension with increased pulmonary blood flow in lambs. Circulation 1995;92:606-13.

13. Wang JR, Zhou Y, Sang K, et al. Association between pulmonary vascular remodeling and expression of hypoxiainducible factor- $1 \alpha$, endothelin- 1 and inducible nitric oxide synthase in pulmonary vessels in neonatal rats with hypoxic pulmonary hypertension. Zhongguo Dang Dai Er Ke Za Zhi 2013;15:138-44.

14. Lehoux S, Lemarié CA, Esposito B, et al. Remodeling pressure-induced matrix metalloproteinase- 9 contributes to early hypertensive. Circulation 2004;109:1041-7.

15. Shimoda LA, Laurie SS. HIF and pulmonary vascular responses to hypoxia. J Appl Physiol 2014;116:867-74.

16. Nossaman BD, Kadowitz PJ. The role of the RhoA/rhokinase pathway in pulmonary hypertension. Curr Drug Discov Technol 2009;6:59-71.

17. Richard DE, Berra E, Pouysségur J. Nonhypoxic pathway mediates the induction of hypoxia-inducible factor $1 \alpha$ in vascular smooth muscle cells. J Biol Chem 2000;275:26765-71.

18. Lauzier MC, Elisabeth L, Maude P, et al. Differential regulation of hypoxia-inducible factor-1 through receptor tyrosine kinase transactivation in vascular smooth muscle cells. Endocrinology 2007;148:4023-31.

19. Zhang R, Wu Y, Zhao M, et al. Role of HIF-1 in the regulation ACE and ACE2 expression in hypoxic human pulmonary artery smooth muscle cells. Am J Physiol Lung Cell Mol Physiol 2009;297:L631-40.

(English Language Editors: R. Scott and J. Chapnick)

Cite this article as: Liu Y, Wen B, Zhang F, Pan XB. Effects of blood oxygen saturation on pulmonary artery remodeling in an in vitro perfusion circuit model. J Thorac Dis 2021;13(4):21692176. doi: $10.21037 / \mathrm{jtd}-20-2124$ 